\title{
Cannabis use predicts shorter duration of untreated psychosis and lower levels of negative symptoms in first-episode psychosis: a South African study
}

\author{
JK Burns', K Jhazbhay', R Emsley² \\ 1Department of Psychiatry, Nelson R. Mandela School of Medicine, University of KwaZulu-Natal, South Africa \\ 2Department of Psychiatry, Faculty of Health Sciences, University of Stellenbosch, Cape Town, South Africa
}

\begin{abstract}
Objective: Cannabis use/abuse is a common co-morbid problem in patients experiencing a first episode of psychotic illness (FEP). The relationship between the clinical presentation of FEP and cannabis abuse is complex and warrants further investigation, especially within the South African context. Method: We tested associations between recent/current cannabis use and duration of untreated psychosis (DUP), age of onset (AO), PANSS-rated (Positive and Negative Syndrome Scale) positive, negative and general psychopathology symptoms and depressive symptoms (Calgary Depression Scale for Schizophrenia) in a sample of 54 patients with FEP. Results: Mean DUP was 34.4 weeks, while mean AO was 24.7 years. Co-morbid cannabis use occurred in 35\% of the sample and was significantly associated with shorter DUP (Mann-Whitney U, $\mathrm{p}=0.026$ ). While not significant, there was also a trend association between cannabis use and lower negative symptoms (Mann-Whitney U, $\mathrm{p}=0.051$ ). Conclusion: Current/recent cannabis use was associated with clinical features of psychosis onset that previously have been associated with better outcome. Medium and long-term outcome for cannabis users however, is likely to depend on whether or not cannabis use is ongoing.
\end{abstract}

Keywords: First-episode psychosis, Cannabis; Duration of untreated psychosis; Age of onset; Symptoms

Received: 30-12-2009

Accepted: 02-03-2010

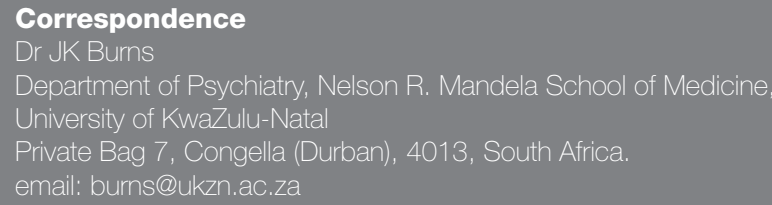

\section{Introduction}

Cannabis use/abuse is a common co-morbid problem in patients experiencing a first episode of psychotic illness. While cannabis use is associated with worse outcome in schizophrenia ${ }^{1-2}$, anecdotal clinical observations suggest that a prominent history of recent cannabis abuse in patients presenting with first-episode psychosis (FEP), predicts rapid resolution of acute symptoms. The relationship then between
\end{abstract}

the clinical presentation of FEP and cannabis abuse is complex and warrants further investigation, especially within the South African context where this pattern of co-morbidity is so prevalent. ${ }^{3}$ It is therefore relevant to explore any associations that may exist between recent/current cannabis use/abuse and clinical features of FEP that previously have been shown to have prognostic value (including age of onset (AO), duration of untreated psychosis (DUP), positive, negative, general psychopathology and depressive symptoms). Importantly, while these features may be predictive of outcome, they are not measures of outcome itself - they might better be considered proxies for outcome.

Sugranyes and colleagues found that cannabis use (irrespective of frequency) was associated with early AO and that $\mathrm{AO}$ decreased as frequency of cannabis use 
increased. ${ }^{4}$ Similarly, González-Pinto and colleagues showed that AO was earlier in cannabis users compared to nonusers, was even earlier in cannabis abusers, and earlier still in those with cannabis dependence. ${ }^{5}$ Regarding symptoms however, FEP patients with a history of cannabis use have less prominent negative symptoms and a predominance of positive symptoms. ${ }^{1,6-7}$ Outcome though is not favourable in patients with co-morbid schizophrenia and cannabis abuse, with evidence indicating more severe and refractory symptoms, poorer treatment-response, higher relapse rates and an overall worse prognosis. ${ }^{1-2}$ A recent study by Baeza and colleagues may illuminate the issue. ${ }^{8}$ At 6 months follow-up, non-cannabis users (NCU) had the worst outcome, while previous cannabis users (PCU) who gave up on commencing treatment had the best outcome. Those cannabis users who were currently using cannabis (CCU) at 6 months had an intermediate outcome. This suggests that in the PCU group, cannabis may have been a major aetiological contributor to psychosis onset - thus, discontinuing cannabis resulted in a favourable outcome. On the other hand the CCU group, who were persisting with cannabis use, remained symptomatic; and their risk of poor long-term outcome is likely to have been high due to their 'dual diagnosis' status. Non-cannabis users (NCU) may have had the worst outcome at 6 months because, in the absence of a major environmental precipitant (cannabis), one might postulate that a greater genetic susceptibility existed. This is relevant to our consideration of cannabis as a risk factor for poor prognosis FEP. One might anticipate that FEP patients with a history of recent or current cannabis use would be more likely in the initial presentation to manifest clinical features of psychosis that have been associated with better outcome in previous studies (with the possible exception of early AO.)

\section{Method}

\section{Participants}

Over a 12 month period, all consecutive patients admitted with FEP to Town Hill Hospital, KwaZulu-Natal Province, South Africa were considered for possible inclusion in the study. Inclusion criteria were: a clinical DSM-IV-TR diagnosis of Schizophreniform Disorder, Schizophrenia and Schizoaffective Disorder; and confirmation of first-episode status through review of clinical records and consultation with the primary caregiver. Exclusion criteria were: age younger than 16 years or older than 45 years; intellectual disability; confirmed history or EEG evidence of epilepsy; evidence of psychotic illness precipitated by a general medical condition; and clear clinical evidence of substanceintoxication or withdrawal (or a definite history of cannabis use within the last week prior to admission). Those meeting inclusion and exclusion criteria were approached and invited to participate. Each participant provided written informed consent after the study was explained in his/her first language. The study was approved by the Biomedical Research Ethics Committee of the University of KwaZuluNatal.

\section{Procedures and instruments}

On admission, patients were interviewed by one of two psychiatrists (JKB or KJ) and rated with the Positive and
Negative Syndrome Scale (PANSS) ${ }^{9}$ as well as the Calgary Depression Scale for Schizophrenia (CDSS). ${ }^{10}$ Both these investigators had received prior training in the administration of these instruments and inter-rater reliability was satisfactory ( $r=0.88$ and 0.84 respectively). Demographic data was recorded by a research nurse including questions about recent or current use of cannabis. Patients were scored as positive for cannabis use if they reported use on a minimum of a weekly basis over the last month prior to admission to hospital.

\section{Definition of clinical features of FEP}

Duration of untreated psychosis (DUP) was defined as the period in weeks between the first appearance of positive psychotic symptoms and the initiation of treatment in hospital. In common with previous studies ${ }^{11}$, onset of psychosis was defined as the presence for at least a week of one or more of the following positive symptoms: hallucinations; delusions; thought disorder; disorganized or bizarre behaviour with a marked deterioration in function. Age of onset (AO) was calculated as the age at initiation of treatment, less the DUP. Positive, negative and general symptoms were derived from the PANSS positive negative and general total scores respectively, while depressive symptoms were derived from the CDSS total score.

\section{Statistical Methods}

Data were analysed using the SPSS version 15.0 (SPSS Inc., Chicago, Illinois) software package and a $\mathrm{P}$ value $<0.05$ was considered statistically significant. Univariate analyses were performed using non-parametric methods due to the non-normal distributions of the dependent variables which were treated as continuous variables. Analysis of variance (ANOVA) methods (Mann-Whitney U) were used with cannabis use dichotomized as the independent variable.

\section{Results}

Fifty four individuals were included in the study with an average age of 25 years and 8 months. The sample was predominantly male (70\%), of Zulu ethnicity (85\%) and of single/separated marital status (85\%) with a mean age of 25 years and 8 months. Cannabis use occurred in 35\% of the sample with a slight (but not significant) male gender bias - 37\% of males and $28 \%$ of females. (Table I)

In terms of clinical features of the first-episode presentation, the mean duration of untreated psychosis (DUP) was 35.08 weeks (median 6 weeks; S.D. 62.01; range: 1-260 weeks), while the mean age of onset of psychosis (AO) was 24.64 years (S.D. 7.6; range: 15-47 years). The mean PANSS positive score was 15.76 (S.D. 6.52; range: 7-32), mean PANSS negative score 13.15 (S.D. 5.68; range: 7-30) and mean PANSS general score 24.85 (S.D. 9.5; range 16-56). The mean CDSS depression score was 6.08 (S.D. 4.83; range: 0-21).

Univariate analysis (Table II) revealed a number of associations between cannabis use and clinical features of FEP. Current or recent cannabis use was significantly associated with shorter DUP (Mann-Whitney U, $p=0.026$ ). Mean DUP for cannabis users was 21.18 weeks (S.D. 48.58) 


\begin{tabular}{|c|c|c|c|c|}
\hline & $n$ & \multicolumn{3}{|l|}{$\%$} \\
\hline $\begin{array}{l}\text { Gender } \\
\text { Male } \\
\text { Female }\end{array}$ & $\begin{array}{l}38 \\
16\end{array}$ & \multicolumn{3}{|l|}{$\begin{array}{l}70 \\
30\end{array}$} \\
\hline $\begin{array}{l}\text { Ethnicity } \\
\text { Zulu } \\
\text { Other }\end{array}$ & $\begin{array}{l}46 \\
8\end{array}$ & \multicolumn{3}{|l|}{$\begin{array}{l}85 \\
15\end{array}$} \\
\hline $\begin{array}{l}\text { Marital status } \\
\text { Single/separated } \\
\text { Married/partner }\end{array}$ & $\begin{array}{l}46 \\
8\end{array}$ & \multicolumn{3}{|l|}{$\begin{array}{l}85 \\
15\end{array}$} \\
\hline $\begin{array}{l}\text { Cannabis } \\
\text { Users } \\
\text { Non-users }\end{array}$ & $\begin{array}{l}17 \\
32\end{array}$ & \multicolumn{3}{|l|}{$\begin{array}{l}35 \\
65\end{array}$} \\
\hline & Mean & S.D. & Median & Min-Max \\
\hline $\begin{array}{l}\text { Age (years) } \\
\text { Age of onset (years) } \\
\text { Duration of untreated psychosis (weeks) } \\
\text { Positive symptom score (PANSS) } \\
\text { Negative symptom score (PANSS) } \\
\text { General psychopathology score (PANSS) } \\
\text { Depressive symptom score (CDSS) }\end{array}$ & $\begin{array}{l}25.8 \\
24.7 \\
35.1 \\
14.2 \\
11.9 \\
18.1 \\
6.1\end{array}$ & $\begin{array}{l}8.1 \\
7.6 \\
62.0 \\
8.1 \\
6.8 \\
12.8 \\
4.8\end{array}$ & $\begin{array}{l}25.0 \\
22.0 \\
6.0 \\
12.5 \\
12.0 \\
12.5 \\
5.0\end{array}$ & $\begin{array}{l}17-48 \\
15-47 \\
1-260 \\
0-32 \\
0-30 \\
0-56 \\
0-21\end{array}$ \\
\hline
\end{tabular}

and for non-users 41.75 weeks (S.D. 67.12). While not significant, there was also a trend association between cannabis use and lower negative symptoms (Mann-Whitney $\mathrm{U}, \mathrm{p}=0.051$ ). The mean PANSS negative score for cannabis users was 8.59 (S.D. 4.96) and for non-users 13.28 (S.D. 6.82). There was no association between cannabis use and AO, positive, general psychopathology or depressive symptoms.

Although it would have been desirable to perform multivariate regression analyses (MVR) on the significant variables in the univariate analysis, we decided not to proceed with MVR due to the small sample size and categorical nature of the independent variable. Under these circumstances the results of a MVR would be of questionable validity.

\section{Discussion}

The ethnic distribution of our sample was consistent with that of the local population while the prevalence of cannabis use/abuse (35\%) approximated that reported in other African studies: 38\% in the Gambia ${ }^{12}$ and 35-49\% in South Africa. ${ }^{3,13}$ The slight gender bias in prevalence of cannabis use observed in our study (37\% of males and $28 \%$ of females) was also similar to that reported by Roos and colleagues. ${ }^{13}$

In contrast to previous studies ${ }^{1,6,14,15}$, we found a significant association between cannabis use and shorter DUP and a trend association between the use of cannabis and a relative absence of negative symptoms. Also, unlike

Table II. Univariate analysis for cannabis use

\begin{tabular}{|c|c|c|c|}
\hline & CANNABIS USERS $(n=17)$ & NON-CANNABIS USERS $(n=32)$ & \\
\hline & Mean (S.D.) & Mean (S.D.) & Significance (MWU) \\
\hline $\begin{array}{l}\text { Duration of Untreated Psychosis (weeks) } \\
\text { Age of onset (years) } \\
\text { Positive symptoms } \\
\text { Negative symptoms } \\
\text { General symptoms } \\
\text { Depressive symptoms }\end{array}$ & $\begin{array}{l}21.18(48.58) \\
22.24(3.51) \\
11.71(6.22) \\
8.59(4.96) \\
14.59(9.38) \\
6.41(4.11)\end{array}$ & $\begin{array}{l}41.75(67.12) \\
26.47(8.91) \\
15.50(8.70) \\
13.28(6.82) \\
19.84(14.81) \\
6.32(5.31)\end{array}$ & $\begin{array}{l}0.026^{\star} \\
0.387 \\
0.628 \\
0.051 \\
0.744 \\
0.862\end{array}$ \\
\hline
\end{tabular}


these studies, we did not find increased positive symptoms, nor was there any association with AO (a finding that has been reported from both developed and developing countries ${ }^{3-5,12-}$ ${ }^{15}$ ). Clearly then the relationship between cannabis use and onset of FEP is complex.

It is possible that the shorter DUP associated with cannabis use in our sample may relate to the specific context within which this study was conducted. Locally produced cannabis within the Province of KwaZulu-Natal is well-known for its very high THC concentration and psychogenic potency ${ }^{16}$; and it is reasonable to speculate that its use may give rise to particularly disruptive symptomatology and behaviour that hastens individuals' pathway to care - thereby shortening DUP.

The association between cannabis use and low or absent negative symptoms has attracted a number of possible explanations. Compton and colleagues argue that individuals with negative symptoms are underrepresented because the apathy, amotivation and social withdrawal associated with negative symptoms impede their ability to access cannabis. ${ }^{1}$ However, we do not find this explanation convincing, especially within the South African context where cannabis is easily accessible. Rather, we favour the suggestion that cannabis may reduce the negative symptoms of psychosis. This notion has received some support, notably from a study by Peralta and Cuesta where low levels of cannabis consumption by patients with schizophrenia attenuated negative symptoms, but had no effect on positive symptoms. ${ }^{17}$ The clinical finding then of lower negative symptoms in FEP patients who use cannabis may reflect self-medicating behaviour as has been suggested by a number of authors ${ }^{17-20}$, but questioned by others. ${ }^{21}$ It is important to note however that the high co-morbidity of cannabis use and psychosis cannot be attributed to self-medication alone. In fact there are now a number of large prospective studies ${ }^{22-24}$ that confirm that primary cannabis use increases risk for subsequent psychotic illness by a factor of two. ${ }^{25}$ The role of cannabis as a risk factor for psychosis must be understood in terms of complex gene-environment interactions where exposure to cannabis modifies gene expression in genetically susceptible individuals. ${ }^{26}$

\section{Strengths and limitations of the study}

Strengths of this study include: the fact that data was obtained through a number of methods including participant and family/caregiver interviews as well as from case notes, thereby enhancing validity; clinical ratings were conducted by trained psychiatrists with good inter-rater reliability and using standardized and validated rating instruments; and the sample was treatment-naive at the time of assessment. Finally, to our knowledge, this is the first study of FEP in a predominantly Zulu sample.

The relatively small sample size is an obvious limitation of the study and together with some missing data for certain variables may have weakened the power of the statistical analysis. In view of this limitation, we elected not to do multivariate analysis (MVA) as we could not be confident of the validity of MVA results. The absence of MVA is obviously a further limitation of the study. Although we relied solely upon self-and caregiver reporting to establish cannabis use, we are satisfied that this is a valid method - Koen and colleaguescompared urine THC testing with self-report of cannabis use and concluded that determination based solely on history is reliable and that THC testing "appears to be of limited value". ${ }^{3}$ Generalization from our results is limited by several factors (some mentioned above) including the fact that ours was an entirely hospital based sample which is likely not to represent all patients in this area. Also, the high rate of HIVseropositivity (22\%) in our sample may be a confounder. In our view however, the potential to confound the results is minimal, as we excluded from the study individuals where a general medical condition was clinically judged to be aetiological of the psychosis. The absence of clinically significant symptoms of HIV-AIDS in our sample suggests that HIV seropositivity is a coincident finding with psychotic disorder (rather than aetiological of the psychosis). Finally, it is important to reiterate that while DUP, AO and symptoms at onset may be predictive of outcome, they are not measures of outcome itself - they might better be considered proxies for outcome.

\section{Conclusion}

With reference then to prognostic features of FEP, it appears that cannabis use in our study is associated with clinical features of psychosis onset that previously have been associated with better outcome, namely shorter DUP and a relative absence of negative symptoms. In terms of a geneenvironment model of psychosis onset, one might postulate that in non-cannabis users, where the contribution of 'environment' is seemingly less, there may be conversely a greater degree of genetic susceptibility (which may be associated with less favourable course and outcome). In the case of cannabis users however, medium and long-term outcome is likely to depend on whether or not cannabis use is ongoing (see discussion on Baeza and colleagues ${ }^{8}$ above), as persistent use is clearly associated with a more continuous illness and a greater predominance of positive symptoms at follow-up. ${ }^{27}$

\section{References}

1. Compton MT, Furman AC, Kaslow NJ. Lower negative symptom scores among cannabis-dependent patients with schizophreniaspectrum disorders: preliminary evidence from an African American first-episode sample. Schizophr Res 2004; 71:61-64.

2. Linszen D, Peters B, de Haan L. Cannabis abuse and the course of schizophrenia. In: Castle DJ, Murray R, eds, Marijuana and Madness. Cambridge: Cambridge University Press, 2004:119-126.

3. Koen L, Jonathan R, Niehaus DJH. Cannabis use and abuse correlates in a homogenous South African schizophrenia population. SAJP 2007; 13(2): 60-66.

4. Sugranyes G, Flamarique I, Parellada E et al. Cannabis use and age of diagnosis of schizophrenia. Eur Psychiatry 2009; 24:282-286.

5. González-Pinto A, Vega P, Ibáñez B et al. Impact of cannabis and other drugs on age at onset of psychosis. J Clin Psychiatry 2008, 69:1210-1216.

6. Van Mastrigt S, Addington J, Addington D. Substance misuse at presentation to an early psychosis program. Soc Psychiatry Psychiatr Epidemiol 2004; 39:69-72.

7. Stirling J, Lewis S, Hopkins R, White C. Cannabis use prior to first onset psychosis predicts spared neurocognition at 10-year followup. Schizophr Res 2005; 75:135-137.

8. Baeza I, Graell M, Moreno D et al. Cannabis use in children and 
adolescents with first-episode psychosis: influence on psychopathology and short-term outcome (CAFEPS study). Schizophr Res 2009; 113:129-137.

9. Kay SR, Fitzbein A, Opler IA. The Positive and Negative Syndrome Scale (PANSS) for schizophrenia. Schizophr Bull 1987; 13:261-267.

10. Addington D, Addington J, Schissel B. A depression rating scale for schizophrenics. Schizophr Res 1990; 3:47-251.

11. Morgan C, Abdul-Al R, Lappin JM, et al. Clinical and social determinants of duration of untreated psychosis in the ÆESOP firstepisode psychosis study. Br J Psychiatry 2006; 189, 446-452.

12. Rolfe M, Tang CM, Sabally S, et al. Psychosis and cannabis abuse in The Gambia: a case-control study. Br J Psychiatry 1993; 163:798801.

13. Roos JL, Pretorius HW, Karayiorgou M, Boraine H. Cannabis and other variables affecting age at onset in a schizophrenia founder population. S Afr Psychiatry Rev 2006; 9:99-103.

14. Talamo, A., Centorrino, F., Tondo, L., et al. Co-morbid substance use in schizophrenia: relation to positive and negative symptoms. Schizophr Res 2006; 86:251-255.

15. Brink S, Oosthuizen P, Emsley R et al. Relationship between substance abuse and first-episode psychosis - a South African perspective. SAJP 2003; 9:7-12.

16. Mail \& Guardian Online. Cannabis abuse in SA twice the global norm. 20 February 2009. http://elections.mg.co.za/story/2009-0220-cannabis-abuse-in-sa-twice-the-global-norm (Accessed 9th December 2009).

17. Peralta V, Cuesta MJ. Influence of cannabis abuse on schizophrenic psychopathology. Acta Psychiatr Scand 1992; 85:127-130.
18. Potvin S, Stip E, Roy JY. Schizophrenia and addiction: an evaluation of the self-medication hypothesis. Encephale 2003; 29:193-203.

19. Goswami S, Mattoo SK, Basu D, Singh G. Substance-abusing schizophrenics: do they self-medicate? Am J Addiction 2004; 13:139-150.

20. Skosnik PD, Spatz-Glenn L, Park S. Cannabis use is associated with schizotypy and attentional disinhibition. Schizophr Res 2001; 48:8392.

21. Verdoux H, Sorbara F, Gindre C et al. Cannabis use and dimensions of psychosis in a nonclinical population of female subjects. Schizophr Res 2003; 59:77-84.

22. Andreasson S, Allebeck P, Engstrom A, Rydberg U. Cannabis and schizophrenia: a longitudinal study of Swedish conscripts. Lancet 1987; 2:1483-1486.

23. van Os J, Bak M, Hanssen $M$ et al. Cannabis and psychosis: a longitudinal population-based study. Am J Epidemiol 2002; 156:319327.

24. Arseneault L, Cannon M, Poulton $R$ et al. Cannabis use in adolescence and risk for adult psychosis: longitudinal prospective study. BMJ 2002; 325:1212-1213.

25. Arseneault L, Cannon M, Witton J, Murray RM. Causal association between cannabis and psychosis: examination of the evidence. $\mathrm{Br} J$ Psychiatry 2004; 184:110-117.

26. Van O., J., B. P. Rutten, et al Gene-environment interactions in schizophrenia: review of epidemiological findings and future directions. Schizophr Bull 2008; 34: 1066-82.

27. Grech A, Van Os J, Jones PB et al. Cannabis use and outcome of recent onset psychosis. Eur Psychiatry 2005; 20:349-353. 